PAPER

\title{
Population based mortality and quality of death certification in progressive supranuclear palsy (Steele-Richardson- Olszewski syndrome)
}

\author{
U Nath, R Thomson, R Wood, Y Ben-Shlomo, A Lees, C Rooney, D Burn
}

J Neurol Neurosurg Psychiatry 2005;76:498-502. doi: 10.1136/jnnp.2004.039370

See end of article for authors' affiliations

Correspondence to:

Dr David J Burn, Regional

Neurosciences Centre, Newcastle General Hospital, Westgate Road Newcastle upon Tyne NE4 6BE, UK; d.j.burn@ncl.ac. uk

Received 16 February 2004 In revised form 9 July 2004 Accepted 27 July 2004
Objective: To estimate the mortality of progressive supranuclear palsy (PSP) and to assess the quality of death certification in patients with PSP who died in England and Wales.

Methods: An analysis was conducted of ICD-9 (International Classification of Diseases, version 9) coded deaths obtained through the Office of National Statistics over an eight year period.

Results: The crude annual mortality rate was 1.77 (95\% confidence interval, 1.64 to 1.90 ) cases per million, using the mid-1996 population estimate for England and Wales. Annual mortality increased over time, possibly as a result of increased incidence or increased awareness of the disorder. Forty nine death certificates from deceased patients previously diagnosed clinically showed that the commonest proximate cause of death was pneumonia, occurring in $45 \%$ of cases (22/49). The underlying cause of death was cited as pneumonia in $14 \%$ of cases (7/49). PSP was mentioned in only $65 \%$ of death certificates (32/49). Eight of the 49 cases (16\%) underwent necropsy and results were available for five of these cases. PSP was confirmed pathologically in four; the remaining case was diagnosed as Parkinson's disease.

Conclusions: Further research is needed to establish the reasons for the observed increase in mortality. Determining the population mortality rate for PSP using the ICD-9 coding system is problematic but is likely to improve following the introduction of ICD-10 updated codes and coding rules.
$\mathrm{T}$ he aetiology, incidence, and outcomes of progressive supranuclear palsy (PSP, Steele-Richardson-Olszewski syndrome), a progressive neurodegenerative disorder with a prevalence in the United Kingdom of five cases per 100000 (95\% confidence interval (CI), 2.5 to 7.5$)^{1}$ are poorly characterised. Clinical features include a variable combination of supranuclear gaze palsy, akinetic-rigid features, early postural instability, axial dystonia, and frontolimbic dementia. ${ }^{2}$ The median disease duration is only five to six years, with bulbar dysfunction leading to aspiration pneumonia, commonly cited as a preterminal event. ${ }^{3}{ }^{4}$ Little is known about the mortality rate for PSP and whether there have been secular changes.

Consensus criteria for the diagnosis of PSP (NINDS-SPSP criteria) divide cases into definite, probable, and possible categories, ${ }^{5}$ with classification of definite PSP requiring pathological confirmation. PSP is most commonly misdiagnosed as Parkinson's disease or cerebrovascular disease. Maher and Lees described the quality of death certification in PSP and reported that only 13 of 30 death certificates $(43.3 \%)$ actually mentioned the disorder. ${ }^{4}$ Eight patients $(26.7 \%)$ were misclassified as having had Parkinson's disease.

Population based mortality estimates in the United Kingdom are based on the underlying cause of death, coded from routine death certificates (table 1). In England and Wales, deaths are registered locally and copies of the register entries sent to the Office for National Statistics (ONS) where they are coded according to the International Classification of Diseases (ICD) system. From 1979 until 2001, deaths were coded using the ninth revision of the ICD (ICD-9). ${ }^{7}$ England and Wales implemented the 10th revision, ICD-10, for mortality coding in $2001 .^{89}$

All conditions mentioned in the death certificate are coded and the underlying cause of death selected. This is done using certain selection and modification rules. ${ }^{6}$ Under the ICD-9 rule 3 system, in cases where the proximate cause of death was pneumonia but the patient had a debilitating neurological illness, the latter was automatically coded as the underlying cause of death only in those death certificates where it was mentioned in Part I of the death certificate.

In ICD-10, rule 3 has been altered so that if a debilitating neurological illness is mentioned in Part II of the death certificate, it would now be coded as the underlying cause of death when the condition mentioned in Part I (for example, pneumonia) is likely to be a terminal event in a person rendered disabled by the condition mentioned in Part II.

The absence of a specific code for PSP in ICD-9 also renders the underlying cause of death problematic, as PSP may be classified under a variety of codes for neurological disorders (table 2). As there is no entry in the index for "progressive supranuclear palsy," the heading "palsy" is taken, and the exact code used is heavily dependent on the precise term written by the certifier, including the order in which the words were written. The closest index entry in ICD-9 for PSP is "supranuclear paralysis" (code 356.8) although confusingly the accompanying textual description is "...hereditary or idiopathic peripheral neuropathy".

In ICD-10, progressive supranuclear ophthalmoplegia and "Steele-Richardson-Olszewski syndrome" are indexed to code G23.1, the tabular list entry for which is "progressive supranuclear ophthalmoplegia." "Progressive supranuclear palsy" is not so indexed, so the ICD-10 index would still code "palsy/paralysis, supranuclear," for which the tabular list term is "motor neurone disease," with an inclusion term for progressive bulbar palsy. There is still no entry for "Palsy, supranuclear progressive" in ICD-10.

In this study, we determined whether the crude and age/ sex standardised population mortality rate for PSP has altered, using a textual analysis of ICD-9 coded information from all death certificates in England and Wales. We have

Abbreviations: NINDS, National Institute of Neurological Disorders and Stroke; ONS, Office of National Statistics; PSP, progressive supranuclear palsy; SPSP, Society for Progressive Supranuclear Palsy 
Table 1 World Health Organisation definitions and death certification practice

1. Proximate cause of death

The condition/s leading directly to death (position la on the death certificate).

2. Underlying cause of death

The disease or injury which initiated the train of events directly leading to death (or the circumstances of the accident or violence which produced the fatal injury).

3. Rule 3 (ICD-9 version)

"If the underlying cause... can be considered a direct sequel to another reported condition, whether in Part I or Part II, select this primary condition."

4. Rule 3 (ICD-10 version)

"If the condition.... is obviously a direct consequence of another reported condition, whether in Part I or Part II, select this primary condition."*

*Although the actual wording is similar, its interpretation has been broadened so that in cases where pneumonia from unspecified organisms would have been the underlying cause of death and another major condition is mentioned elsewhere in the certificate, the latter will be selected as the underlying cause of death, whether or not a causal sequence can be presumed. ${ }^{6}$ It is to be assumed that conditions which are wasting or paralysing, compromised immunity, infectious diseases, other respiratory diseases, and any major injury or operation may be the underlying cause of these types of pneumonia.

ICD, International Classification of Diseases.

also examined how this rate will change under the new coding rules for ICD-10. Finally, we also assessed the quality of death certification in a cohort of patients with a known prior clinical diagnosis of PSP.

\section{METHODS}

Mortality

We identified deaths from PSP between 1 January 1993 and 31 December 2000 from the Office of National Statistics (ONS) central register. The search strategy used the ICD-9 system and consisted of several steps, illustrated in the flow chart in fig 1.

(1) Data were extracted using several codes recommended by the ONS as being most likely to identify cases of PSP (table 2). In addition we obtained death certificates that included a diagnosis of Parkinson's disease, previously shown to be a source of misclassification of PSP. ${ }^{4}$

(2) We hand searched the file of full text death certification data to identify cases of PSP in the files containing deaths coded (in any position) for progressive supranuclear palsy or Steele-Richardson-Olszewski syndrome.

(3) We then constructed and tested text based computerised search strategies in an MS access database. Search terms were then chosen-for example, "rich", "steele"—and tested upon a small proportion of the data in order to

Table 2 ICD-9 codes used for identification of cases of deaths from progressive supranuclear palsy

\begin{tabular}{|c|c|}
\hline ICD-9 code & Textual classification \\
\hline 344.8 & $\begin{array}{l}\text { Palsy } \\
\text { Bulbar } \\
\text { Supranuclear not elsewhere classified }\end{array}$ \\
\hline 333.0 & $\begin{array}{l}\text { Other diseases of basal ganglia } \\
\text { Progressive supranuclear ophthalmoplegia }\end{array}$ \\
\hline 332.0 & Parkinson's disease \\
\hline 335.2 & $\begin{array}{l}\text { Palsy/paralysis } \\
\text { Bulbar } \\
\text { Progressive }\end{array}$ \\
\hline 356.8 & $\begin{array}{l}\text { Paralysis } \\
\text { Supranuclear }\end{array}$ \\
\hline
\end{tabular}

ICD-9, International Classification of Diseases, version 9. assess which identified the greatest number of cases of PSP with the least number of errors (false positives). The most effective strategy was a text based search strategy which searched for the terms "supra," "rich," "pals," or "nucle." This strategy was found to be superior to the manual strategy (point (2)) which was therefore abandoned.

(4) False positives were excluded from further analysis. We then reviewed all the remaining cases in order to assign cause of death manually using the ONS coding rules.

The crude mortality was calculated using mid-year population estimates, based upon various criteria for assigning PSP as the cause of death. First, the mortality was calculated assigning PSP as the underlying cause of death whenever present, regardless of its position on the death certificate. Second, we reassigned underlying cause of death using different versions of rule 3, before and after implementation of ICD-10 in 2001. Trend analysis was undertaken using the $\chi^{2}$ statistic ( $1 \mathrm{df}$ ) for PSP deaths in each group. We also used indirect age/sex standardisation of the annual mortality rates using the whole study period as the standard population to calculate standard mortality ratios and an age/ sex adjusted mortality rate for each year. Lastly we used Poisson regression analysis to calculate the risk ratios (95\% confidence intervals (CI) and p values) for sex and time period (1993 to 1996, 1997 to 2000) having adjusted for differences in the age distribution.

\section{Quality of death certification}

We previously reported a study to estimate the prevalence of PSP in the United Kingdom. ${ }^{1}$ Patients were identified by retrospective case record review as suffering from either probable or possible PSP, according to NINDS-SPSP criteria. Patients were included in the prevalence estimate if they were alive and resident in the United Kingdom on 1 January 1999. All available death certificates for patients from this cohort in England and Wales who died between 1 January 1999 and 31 December 2000 were obtained from the ONS and reviewed to assess their quality and completeness.

Ethical approval was obtained from the Northern and Yorkshire multicentre research and ethics committee (MREC) and UK local research ethics committees.

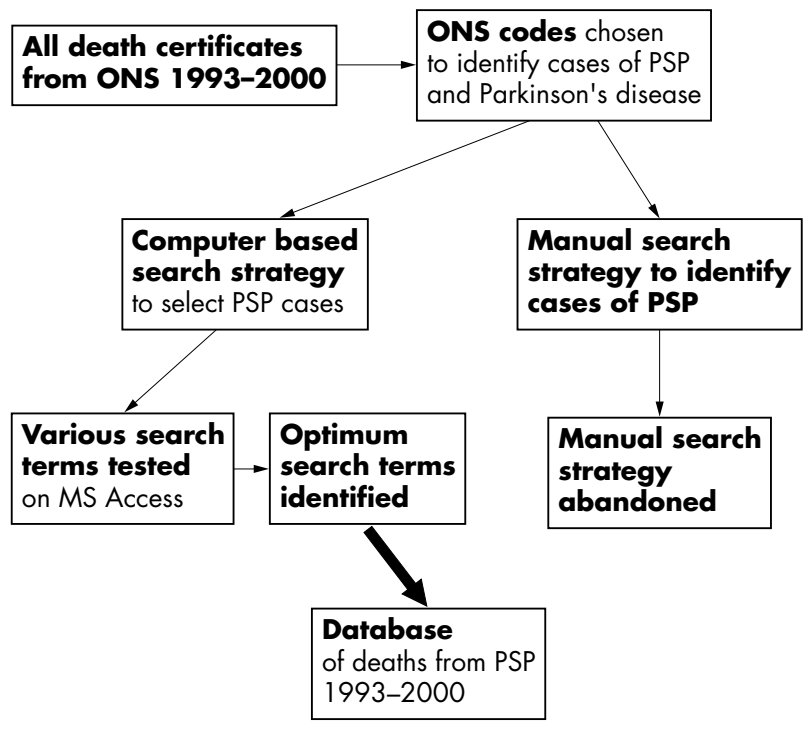

Figure 1 Algorithm of search strategies to identify deaths from progressive supranuclear palsy from 1 January 1993 to 31 December 2000. 


\section{RESULTS}

We identified 71408 death certificates during the eight year period 1993 to 2000 by means of an automated search for five specified ICD-9 codes (table 2). Using the search terms "supra," "rich," "pals," and "nucle," 749 cases were thus identified, of which 23 were erroneous-for example, "supracondylar fracture of the humerus" - and one was found to be from 1992. These cases were excluded from further analysis. In the 725 remaining cases, PSP was cited as a textual diagnosis in at least one position on the death certificate, yielding an average of 90 deaths a year in patients with PSP. Using the mid-1996 population estimate for England and Wales, this provides a crude annual mortality for this period of 1.77 (95\% CI, 1.64 to 1.90 ) cases per million. The number of death certificates mentioning PSP rose annually (table 3, p value for trend $<0.0001$ ). The age specific death rates dividing data into groups (years 1993 to 1996 and years 1998 to 2000) are given in fig 2 , showing an increase with age, which peaks in the 75 to 79 age group and is greater for the later time period.

The number of cases where the underlying cause of death according to ICD-9 and ICD-10 interpretations of rule 3 was also assessed and the revised annual mortality calculated. Of the 725 cases, 539 would have been assigned PSP as the underlying cause of death using the earlier version of rule 3, giving a crude population annual mortality of 1.31 (95\% CI, 1.20 to 1.43 ) per million. Using the later version of rule 3, 641 cases would have been assigned PSP as the underlying cause of death, resulting in a higher crude population annual mortality of 1.56 (1.44 to 1.69) per million. To examine whether the increase in mortality over time could be explained by demographic changes, we calculated the standardised mortality rates (table 4 ) which showed a more than twofold increase between 1993 and 2000, which is not explained by changes in the age and sex structure of the population. This was confirmed by the Poission regression estimates that found that women were $41 \%$ less likely to have a death certificate with PSP (risk ratio 0.59 (0.51 to 0.68), $\mathrm{p}<0.001$ ) and that mortality had almost doubled for the second time period (risk ratio 1.83 ( 1.57 to 2.14 ), $\mathrm{p}<0.001$ ).

Forty nine deaths within England and Wales occurred in a separate cohort of 187 PSP cases in the United Kingdom between I January 1999 and 31 December 2000. ${ }^{1}$ Death certificates were traced for each of these cases. Twenty six deceased cases were male and 23 were female. Twenty cases had been classified as "possible" and 29 as "probable" PSP before death. PSP was mentioned in 32 of the 49 death certificates $(65 \%)$. There were no significant differences in sex, median age, or place of death (home or institution) between cases where PSP was stated on the certificate and in those where it was not.

The commonest proximate cause of death was pneumonia, occurring in 22 of the 49 cases (45\%). The chosen ICD- 9 codes

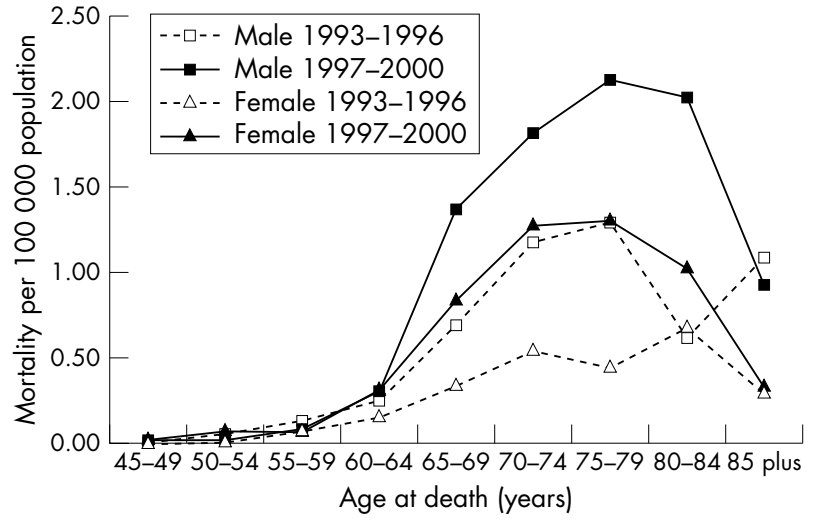

Figure 2 Age specific mortality rates from progressive supranuclear palsy in England and Wales for 1993 to 1996 and 1997 to 2000 by sex.

(table 2) would only have identified 25 of the 49 cases. Of known cases, $31 \%$ (15 of 49 ) had the underlying cause of death assigned correctly as "paralysis, supranuclear." Four per cent of cases were coded as having Parkinson's disease as the underlying cause of death. The underlying cause of death was coded as a pneumonic illness in $14 \%$ of cases. PSP was mentioned in Part II of the death certificate in two of these cases; three of five cases where PSP had not been mentioned were given a (mis)diagnosis (two Parkinson's disease and one of multiple system atrophy). In the remaining two cases, bronchopneumonia was the sole entry on the death certificate. Eight of the 49 cases (16\%) underwent necropsy. Pathological data were available for five cases. PSP was confirmed in four of these five cases. The remaining case, previously classified as possible PSP, had a pathological diagnosis of Parkinson's disease.

\section{DISCUSSION}

Our assessment of the annual population mortality from PSP revealed evidence of increasing mortality over time in the United Kingdom, which was unlikely to be by chance. The crude annual mortality using different methods ranged from 1.31 ( 1.20 to 1.43 ) to 1.77 ( 1.64 to 1.90 ) cases per million. Mortality increased with age for both men and women, peaking at ages 75 to 79 years. This may reflect the incidence pattern, given the uniformly poor survival for all ages. Alternatively, other competing causes of death may lower the apparent mortality in the older age groups or this may reflect age related differences in diagnosis or certification, so that PSP in patients over 79 years is underdiagnosed. Indirectly, age/sex standardised rates confirm that the observed increase in mortality is not an artefact of the changing age/sex structure in the underlying population.

Table 3 Deaths from progressive supranuclear palsy, 1993 to 2000

\begin{tabular}{|c|c|c|c|c|c|}
\hline Year & $\begin{array}{l}\text { Deaths (five } \\
\text { specified ICD-9 } \\
\text { codes) }\end{array}$ & $\begin{array}{l}\text { E\&W pop'n } \\
\text { mid-year estimate }\end{array}$ & $\begin{array}{l}\text { Mortality per million (PSP } \\
\text { anywhere in certificate) }\end{array}$ & $\begin{array}{l}\text { Mortality per million } \\
\text { (ICD-9 rule 3) }\end{array}$ & $\begin{array}{l}\text { Mortality per million } \\
\text { (ICD-10 rule 3) }\end{array}$ \\
\hline 1993 & 9263 & 50944500 & 1.08 & 0.77 & 0.92 \\
\hline 1994 & 8773 & 51051500 & 0.96 & 0.61 & 0.82 \\
\hline 1995 & 9114 & 51175200 & 1.47 & 1.09 & 1.25 \\
\hline 1996 & 8744 & 51289100 & 1.44 & 1.17 & 1.35 \\
\hline 1997 & 8929 & 51412600 & 1.96 & 1.42 & 1.79 \\
\hline 1998 & 8887 & 51550400 & 2.15 & 1.63 & 1.88 \\
\hline 1999 & 9062 & 51730100 & 2.34 & 2.01 & 2.17 \\
\hline 2000 & 8636 & 51897300 & 2.68 & 1.77 & 2.27 \\
\hline Total & 71408 & 51289100 & $\begin{array}{l}1.77(95 \% \mathrm{Cl}, 1.64 \text { to } 1.90) \\
(p<0.0001)\end{array}$ & $\begin{array}{l}1.31(95 \% \mathrm{Cl}, 1.20 \text { to } 1.43) \\
(\mathrm{p}<0.0001)\end{array}$ & $\begin{array}{l}1.56(95 \% \mathrm{Cl} 1.44 \text { to } 1.69) \\
(\mathrm{p}<0.0001)\end{array}$ \\
\hline
\end{tabular}

E\&W, England and Wales; ICD, International Classification of Diseases; pop'n, population; PSP, progressive supranuclear palsy. 
Table 4 Indirect standardised mortality rates from 1993 to 2000

\begin{tabular}{lrrrrrrr}
\hline Year & 1993 & 1994 & 1995 & 1996 & 1997 & 1998 & 1999 \\
\hline Total expected deaths & 89.70 & 89.81 & 90.24 & 90.63 & 91.07 & 91.64 & 92.02 \\
Actual deaths & 55.00 & 49.00 & 75.00 & 74.00 & 101.00 & 111.00 & 121.00 \\
SMR & 0.61 & 0.55 & 0.83 & 0.82 & 1.11 & 1.21 & 1.31 \\
Indirect standardised age/sex & 1.10 & 0.98 & 1.50 & 1.47 & 2.00 & 2.18 & 2.37 \\
$\begin{array}{l}\text { adjusted mortality rate } \\
\text { (per million population) }\end{array}$ & & & & & & & \\
\hline
\end{tabular}

SMR, standardised mortality ratio.

The study had certain limitations, underestimating the true rates. Our study was unable to identify cases of PSP where the diagnosis was not mentioned on the death certificate. This is clinically appropriate if the cause of death was unrelated to PSP-for example, disseminated carcinomatosis. However, as we observed with the death certificates from our cohort, on some occasions the cause of death was cited as bronchopneumonia with no mention of PSP on the death certificate. Such cases are impossible to identify without a necropsy diagnosis. PSP may also have been misclassified under a wider range of codes than we used. No single search term was able to produce an effective automated screening of death certificates with a textual diagnosis of PSP. We were also unable to either clinically confirm the diagnosis of PSP or identify the true underlying cause of death (if different) by case record review.

More accurate data for population mortality from PSP may be available from 2001 onwards in the United Kingdom, following the introduction of ICD-10. The mortality rate calculated using only cases where PSP could be assigned as the underlying cause of death was lower using the ICD-9 coding rules than with ICD-10. A Swedish study designed to analyse the effect of a similar change in coding systems reported a $20 \%$ fall in deaths assigned to any pneumonic illness, while deaths attributable to a neurological disorder rose. ${ }^{10}$ The broader reinterpretation of coding rules in the ICD-10 version therefore improves classification where the underlying cause of death is PSP but it cannot correct the underascertainment of cases of miscertification. Future studies will be required to confirm the impact of both ICD10 changes to coding and classification.

The crude annual mortality from PSP increased from 1.08 to 2.68 per million over the eight year study period. As chance is unlikely to explain this trend, various other explanations must be considered. Increased interest in neurodegenerative disorders may have resulted in increased physician awareness of the disorder and hence increased notification. Changes in clinical criteria during the investigating period, potentially increasing diagnostic sensitivity, may also have contributed. ${ }^{5}$ Although the proportion of older people in the United Kingdom is increasing, this cannot explain the marked secular trend, as this was still seen after accounting for these changes. It is possible that there has been a true increase in observed deaths from PSP. It is unlikely that the disease is becoming increasingly aggressive or management is less effective. More likely is the possibility that improved health care in general reduces the mortality from other competing diseases, such as myocardial infarction, so that patients with PSP are more likely to die from the PSP itself than from another cause. Finally, there may be a true increase in the incidence of PSP. Further studies are required to confirm or refute these hypotheses.

We were able to review the records of a small proportion of patients previously included in a prevalence study. ${ }^{1}$ Despite the diagnosis having been made before death in all these cases, PSP was mentioned in the text of the death certificate in only $65 \%$. Nevertheless, this represents an improvement compared with a previous similar study, ${ }^{4}$ where the diagnosis of PSP was only mentioned in $43 \%$ of cases ( $22 \%$ increase $(95 \%$ CI, $0 \%$ to $44 \%), p=0.06$ ). In the present study, pneumonia was the most common proximate cause of death, occurring in $45 \%$ of 49 cases. For seven cases ( $14 \%$ ), the underlying cause of death was coded as pneumonia, while PSP was not mentioned in five of these seven death certificates. Three cases were misdiagnosed as having another form of parkinsonism and in the remaining two cases, bronchopneumonia was the sole entry on the death certificate.

Necropsy results for only five of the eight cases who underwent necropsy examination were available, even though a definitive diagnosis of PSP requires pathological confirmation. ${ }^{5}$ The importance of the latter is highlighted by the fact that one case in our study, thought to have possible PSP, was found at necropsy to have Parkinson's disease.

In conclusion, this study is the first to review death certification data systematically to address mortality from PSP in the United Kingdom. It suggests there is an increasing mortality, though why this should be is unclear. Were this to reflect an increasing incidence, it would support the role of environmental factors in the aetiology of PSP, which remains unknown. ${ }^{11}{ }^{12}$ There is a need for further monitoring of mortality trends, which will be easier with the introduction of ICD-10. Ultimately only standardised population based screening studies, with incidence data over time, will determine if this disease is truly becoming more common rather than simply being more frequently diagnosed.

\section{ACKNOWLEDGEMENTS}

UN was supported by a grant from the PSP (Europe) Association. We wish to thank Ms P Riach, Ms G Brackett, Mr J White, Dr L Cook from the Office of National Statistics, Dr S Daniel of the UK Parkinson's Disease Brain Bank, and all colleagues who referred cases both nationally and regionally, for their assistance with this study.

\section{Authors' affiliations}

U Nath, D Burn, Department of Neurology, University of Newcastle upon Tyne, Newcastle upon Tyne, UK

R Thomson, R Wood, School of Population and Health Sciences (Epidemiology and Public Health), University of Newcastle upon Tyne Y Ben-Shlomo, Department of Social Medicine, Bristol University, Bristol, UK

A Lees, Sara Koe PSP Centre and Institute of Neurology, Queen Square, London $\mathrm{WCl}$, UK

C Rooney, Office of National Statistics, London, UK

Competing interests: none declared

\section{REFERENCES}

1 Nath U, Ben-Shlomo Y, Thomson R, et al. The prevalence of progressive supranuclear palsy (Steele-Richardson-Olszewski syndrome) in the UK. Brain 2001; 124:1438-49.

2 Steele J, Richardson C, Olszewski J. Progressive supranuclear palsy. Arch Neurol 1964; 10:333-59.

3 Brusa A, Mancardi G, Buguini O. Progressive supranuclear palsy. Ital J Neurol Sci 1980;4:205-22.

4 Maher E, Lees A. The clinical features and natural history of PSP. Neurology 1986;36:1005-8. 
5 Litvan I, Agid Y, Calne D. Clinical research criteria for the diagnosis of progressive supranuclear palsy (Steele-Richardson-Olszewski syndrome): report of the NINDS-SPSP international workshop. Neurology 1996;47:1-9.

6 Rooney C, Devis T. Mortality trends by cause of death in England and Wales 1980-94: the impact of introducing automated cause coding and related changes in 1993. Popul Trends 1996;86:29-35.

7 World Health Organisation. International classification of diseases, 9th revision. Geneva: WHO, 1977.

8 Rooney C, Smith S. Implementation of ICD-10 for mortality data in England and Wales from January 2001. Health Stat Q 2000;8:41-50.
9 World Health Organisation. International statistical classification of diseases and related health problems, 10th revision. Geneva: WHO, 1992.

10 Johannson L. Swedish Bridge-Coding Study, ICD9-ICD10: design and preliminary results. Proceedings of the International Collaborative Effort on Automating Mortality Statistics (in press).

11 Nath U, Burn D. Epidemiology of progressive supranuclear palsy (SteeleRichardson-Olszewski syndrome). Parkinsonism Relat Disord 2000;6: 145-53.

12 Zermansky A, Ben-Shlomo Y. Epidemiological aspects. In: Litvan I, ed. Atypical parkinsonian disorders: clinical and research aspects, New Jersey: Humana Press (in press). 